\title{
ENVIO DIRETO DE PELOTAS USINA 8 (VALE) PARA ABASTECIMENTO DE ALTO FORNOS NA ARCELOR MITTAL TUBARÃO*
}

Fabio Dummer ${ }^{1}$ Graciely Koepp ${ }^{2}$ Josiane Batista ${ }^{3}$ Juliana Mendes ${ }^{4}$

\section{Resumo}

Este trabalho apresenta o desempenho de duas grandes empresas, após a implantação do envio direto de pelotas da Usina de Pelotização Vale 8 para abastecimento dos Alto Fornos da Arcelor Mittal Tubarão, com o propósito de aumentar a produtividade, reduzir custos, otimizando as rotinas operacionais e a utilização da mão de obra. Esse processo é pioneiro no atendimento a clientes de fornecimento de pelotas.

Palavras-chave: Alto forno; Processo de pelotização; Atendimento ao cliente.

\section{DIRECT PELLETS DIRECTLY FROM PELLETIZING 8 TO ARCELOR MITTAL TUBARÃO BLAST FURNACES}

\begin{abstract}
This work presents the performance of two large companies, with the direct pellets directly from Pelletizing 8 to Arcelor Mittal Tubarão blast furnaces, with the purpose of increasing productivity, reducing costs, optimizing operational routines and optimization of manpower. This process is pioneer in serving clients in the supply of pellets.
\end{abstract}

Keywords: Blast furnace; Pelletizing process; Customer service.

Engenheiro de produção, Coordenador Abastecimento Minérios AMT, Vitoria, ES, Brasil.

Engenheiro de Produção, Supervisora de Garantia de Qualidade, VALE, Vitoria, ES, Brasil.

Engenheiro de Produção, Engenheiro Sênior, VALE, Vitoria, ES, Brasil.

Administrador, Analista, DIPE, VALE, Vitoria, ES, Brasil. 


\section{INTRODUÇÃO}

Após o start up da Usina de Pelotização Vale 8, surgiram dificuldades de retirada de pelotas do pátio de estocagem.

Única rota de transferência era para o Porto de Tubarão e a mesma apresentava baixa confiabilidade e calendários de manutenção descasados, dificultando assim embarques/carregamentos das pelotas.

Com o surgimento de dificuldades, oportunidades de melhorias surgiram em parceria com cliente: envio direto de pelotas Usina 8 para AMT. Uma nova oportunidade de transferência de pelotas.

O que é envio direto?

Consiste no envio direto de pelotas produzidas na Usina 8 para consumo em alto fornos do cliente AMT, sem necessidade de empilhamento no pátio de estocagem da usina e do cliente.

Um fluxograma demonstrando o envio direto é apresentado na figura [1-2].

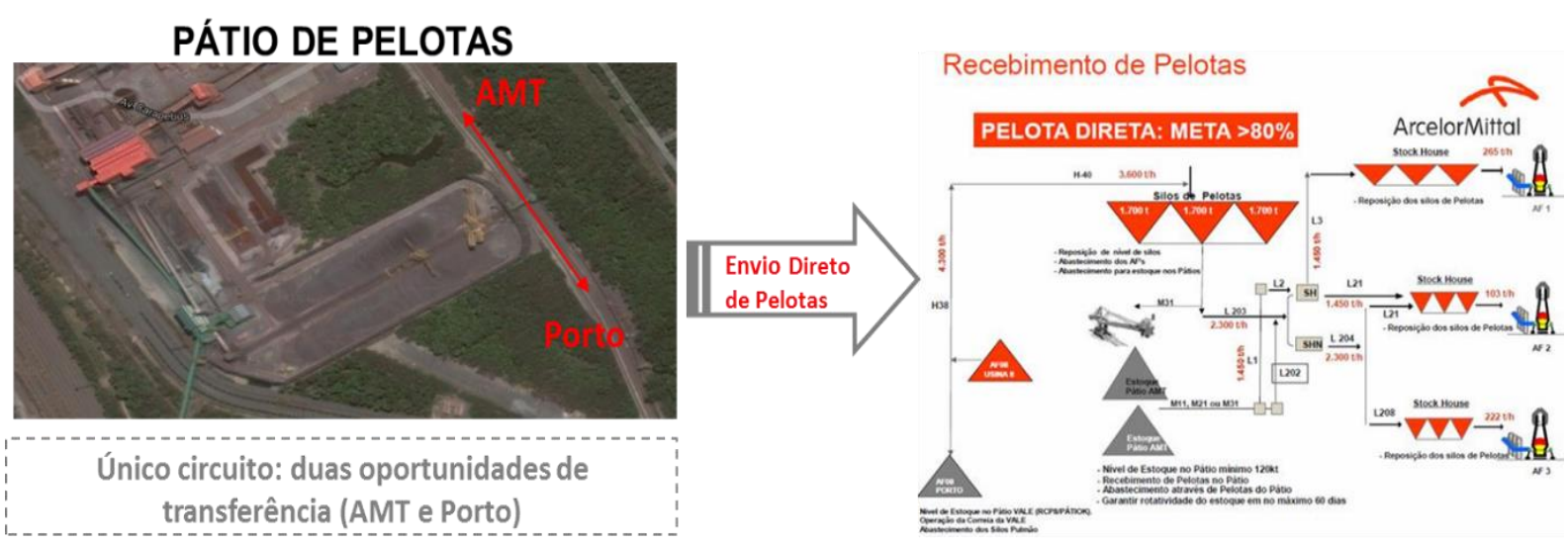

O atual nível competitivo mundial tem feito empresas dos mais variados segmentos buscarem aumentar sua competitividade utilizando estratégias que valorizem atributos como custos e qualidade [3].

A estratégia do envio direto de pelotas enfatiza o aprimoramento da relação cliente e fornecedor, pois surge um novo paradigma de aquisição que impõe a necessidade de se estabelecer regimes de parceria, como forma de se obter vantagens competitivas. Nessa relação de parceria, comprador e fornecedor trabalham em conjunto, predominando a cooperação e o compartilhamento. O relacionamento é, neste caso, planejado e contínuo, baseado na confiança mútua e no planejamento conjunto, além de assistência e visitas mútuas [4].

Para criação desta nova rotina de fornecimento, parâmetros de qualidade do produto foram definidos, flexibilizando os mesmos, já que o consumo da pelota será imediato, sem necessidade de movimentação das mesmas com máquinas (empilhadeiras e recuperadoras) e estocagem, eliminando a degradação do produto.

Também foi criada rotina mensal de reuniões entre VALE e AMT para definição de calendário integrado de manutenção. Pois com o envio direto uma parada da rota de abastecimento deve ser bem planejada. 


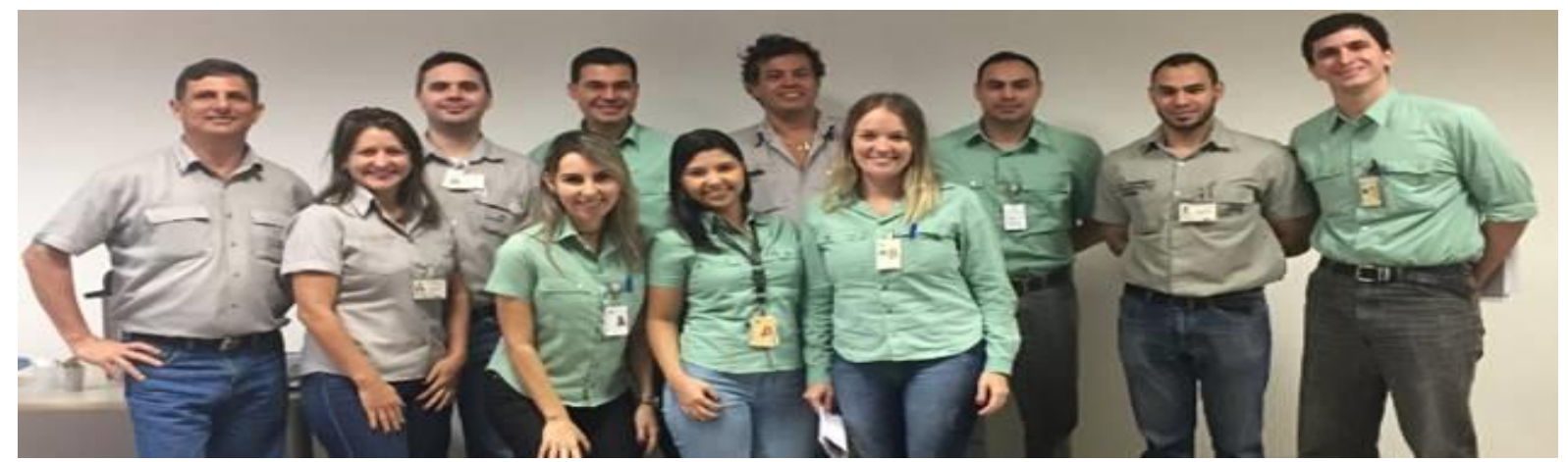

Figura 2. Registro de Reunião de Planejamento Integrado de Manutenção VALE e AMT

Outro fator importante verificado com o envio direto de pelotas Vale para alto fornos AMT foi a redução de custos nas operações das duas empresas, fator altamente positivo. Pois, no ambiente da globalização, a gestão estratégica dos custos surge como uma resposta para atender às demandas do sistema econômico com relação às novas perspectivas e alternativas proporcionadas pela remodelação dos mercados e um contexto de busca por melhoria continua [5].

\section{MATERIAIS E MÉTODOS}

Como mencionado anteriormente, o objetivo deste trabalho foi aumentar a produtividade, reduzir movimentação de pelotas com máquinas e necessidade de estocagem das mesmas. Além reduzir custos, otimizando as rotinas operacionais e a utilização da mão de obra.

Após a decisão da implantação do envio direto de pelotas Usina 8 para AMT, foi definido um macro cronograma para que esta inovação acontecesse:

1. Planejamento da implantação da inovação no envio de pelotas para clientes

2. Levantamento de variáveis críticas de qualidade do produto e restrições de envio

3. Definição dos parâmetros de qualidade e limites de corte do envio de pelotas quando os mesmos não forem atendidos

4. Planejamento da amostragem do produto e rotina de envio dos ensaios realizados para o cliente

5. Definição de indicadores de performance

6. Treinamento inicial com toda equipe Vale e AMT sobre nova forma de envio de pelotas (Supervisores, Engenheiros, Técnicos).

7. Padronização / Análise do processo

8. Fechamento

Todo planejamento e execução foi acompanhado através de plano de ação e definido período para operação assistida com acompanhamento gerencial em ambas empresas.

\section{RESULTADOS E DISCUSSÃO}

Com a implantação do envio direto foram identificadas vantagens tanto para 0 cliente, quanto para o fornecedor.

Resultados obtidos no fornecedor: 
- Com o compromisso do envio direto firmado com o cliente, surgiu o desafio de garantir maior confiabilidade da rota de abastecimento e como produto foi construído Calendário Integrado de Manutenções Vale \& AMT;

\begin{tabular}{|c|c|c|c|c|c|c|c|c|c|c|}
\hline & Circuito & Tempo & Início & Fim & JUL & AGO & SET & OUT & NOV & DEZ \\
\hline \multirow{3}{*}{ Porto } & H38 & $112 \mathrm{~h}$ & 28/ago & $01 /$ set & & $112 \mathrm{~h}$ & & & $48 \mathrm{~h}$ & \\
\hline & $8 \mathrm{PP} 10$ & $48 \mathrm{~h}$ & & & $48 \mathrm{~h}$ & & & & & \\
\hline & RCP9 & $144 \mathrm{~h}$ & 28/ago & $03 /$ set & & 144h & & & & \\
\hline Usina & Parada Usina 8 & $86 h$ & $28 / 200$ & $01 / 5 e t$ & & $86 \mathrm{~h}$ & & & & \\
\hline \multirow{2}{*}{ AMT } & \begin{tabular}{|c|} 
Circuito \\
\end{tabular} & $36 \mathrm{~h}$ & \multicolumn{2}{|c|}{ Rotina a cada 60 dias } & $36 \mathrm{~h}$ & & $36 \mathrm{~h}$ & & $36 \mathrm{~h}$ & \\
\hline & Parada Sinter & $216 \mathrm{~h}$ & $28 / \mathrm{jul}$ & $05 / \mathrm{ago}$ & $216 \mathrm{~h}$ & & & $24 \mathrm{~h}$ & & \\
\hline
\end{tabular}

Figura 3. Calendário Integrado de Manutenção VALE e AMT

- Eliminação do gargalo de saída de pelotas da Usina 8, através de melhor gestão de pátio;

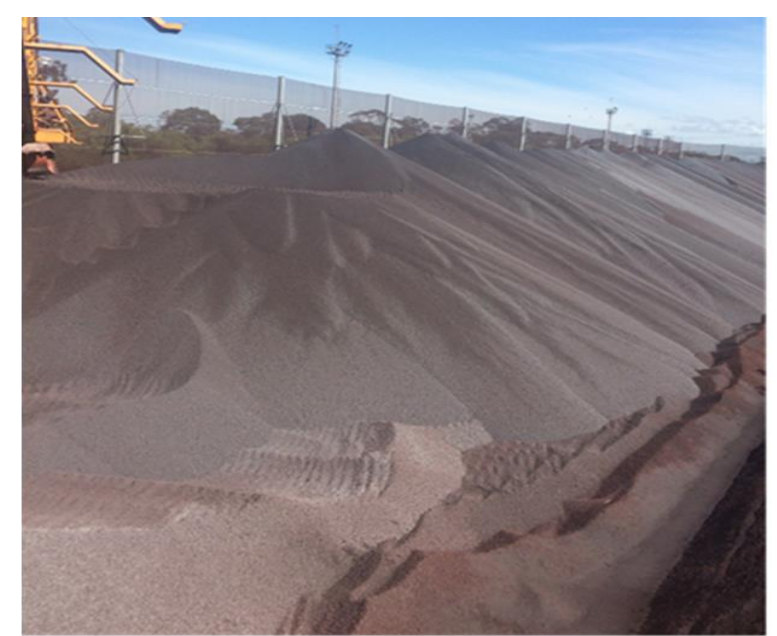

Antes : pátio cheio com risco de parada de usina por falta de área de estocagem

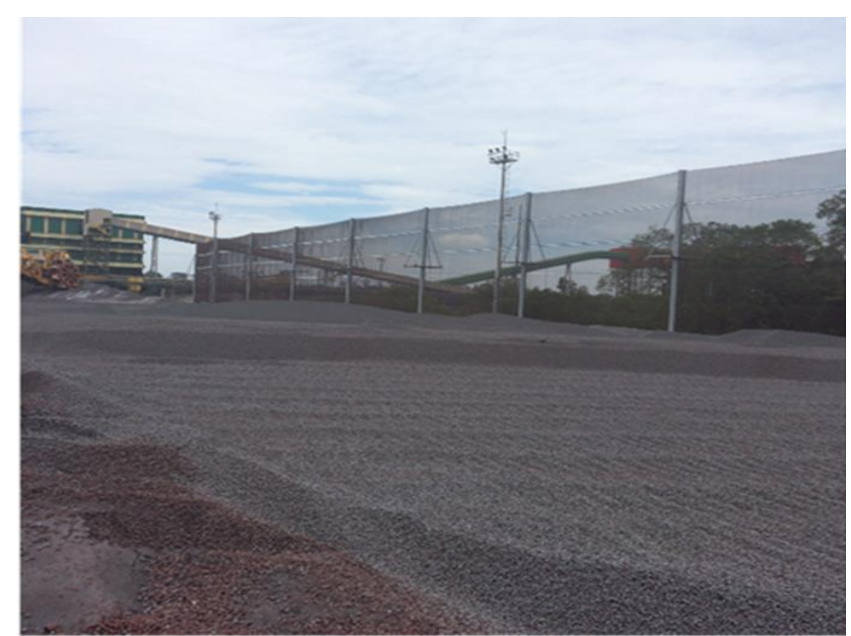

Depois : gestão adequada dos pátios de estocagem

Figura 4. Gestão Pátio de Estocagem Usina 8

- Flexibilização da qualidade do produto enviado com o benefício do envio direto (sem empilhamento e recuperação). Desta forma, é possível absorver maiores variações do processo produtivo da usina;

- Ganho de faturamento.

Resultados obtidos no fornecedor:

- Maior estabilidade no processo produtivo, devido a redução da umidade e \% finos da pelota fornecida. 


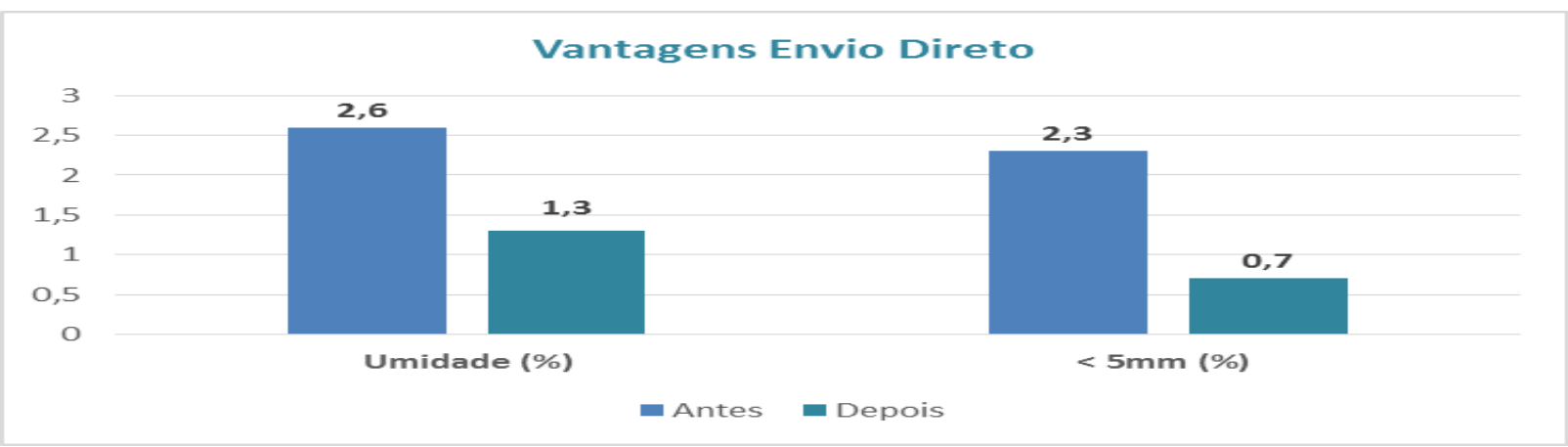

Figura 5. Comparativo dos resultados de umidade e $<5 \mathrm{~mm}$ do produto fornecido (antes e depois)

- O envio direto de pelotas faz parte do plano estratégico AMT até 2020 e garantiu o atendimento de desafios na performance operacional do cliente. Após sua implantação em 2015, foi possível atingir a meta de Coke Rate.

\subsection{Padronização}

Com a implantação do envio direto de pelotas, foram definidos critérios para corte de envio ao cliente devido a parâmetros de qualidade fora da especificação definida.

\begin{tabular}{|c|c|c|c|}
\hline Parâmetro & $\begin{array}{c}\text { Limite } \\
\text { Contratual }\end{array}$ & $\begin{array}{c}\text { Tolerância para } \\
\text { solicitar especial e } \\
\text { aguardar confirmação }\end{array}$ & $\begin{array}{c}\text { Tolerância para } \\
\text { corte imediato do } \\
\text { envio direto }\end{array}$ \\
\hline Basicidade & 0,95 a 1,15 & $\begin{array}{c}\text { Maior que 1,15 } \\
\text { Menor que 1,00 }\end{array}$ & Maior que 1,20 \\
Menor que 0,95
\end{tabular}

\footnotetext{
Obs.: Se for necessário cortar o abastecimento devido qualidade, o resultado de qualidade que justificou a interrupçăo deverá também ser informado ao cliente e inserir no início do email a seguinte informação adequando para o parâmetro que houver necessidade:

Informo que foi realizado o corte do envio da produção devido queda da Bas2.

Somente para caso de corte imediato que a informação será adicionada ao e-mail.
}

Figura 6. Procedimento Envio Direto

\section{CONCLUSÃO}

Através da implantação deste projeto pioneiro no atendimento a clientes de fornecimento de pelotas, muitas vantagens foram identificadas no fornecedor (Vale) como no cliente (AMT), conforme descrito acima. Os objetivos de aumentar a produtividade, reduzir movimentação de pelotas com máquinas e necessidade de estocagem das mesmas. Além reduzir custos, otimizando as rotinas operacionais e a utilização da mão de obra foram atingidos. O processo de envio direto de pelotas da Usina 8 (VALE) para abastecimento dos alto fornos da AMT é uma realidade estabelecida através de grande parceria entre as duas empresas. 


\section{REFERÊNCIAS}

1 Manual de Operação das Usinas de Pelotização da Vale.

2 Meyer K. Pelletizing of Iron Ores. Springer-Verlag Berlin, 302 p, 1980.

3 Shimizu F, Kazumi U Basso LFC; Nakamura WT. Produção enxuta e desempenho de mercado: uma análise para o setor de máquinas e implementos agrícolas no Brasil. In: Simpósio de Administração da produção, logística e operações internacionais (Simpoi), 9,2006, São Paulo. Anais... São Paulo: FGV-EAESP,

4 Portal Educação, Google. Disponível em: https://www.portaleducacao.com.br > Home > Artigos > Administração e Gestão. Acesso em 01 Junho de 2017. 